\title{
ENDOGENOUS DOWNWARD JUMP DIFFUSION AND BLOW UP PHENOMENA BEFORE CRASH
}

\author{
Youngmee Kwon, Intae Jeon, and Hye-Jeong Kang
}

\begin{abstract}
We consider jump processes which has only downward jumps with size a fixed fraction of the current process. The jumps of the processes are interpreted as crashes and we assume that the jump intensity is a nondecreasing function of the current process say $\lambda(X)(X=X(t)$ : process).

For the case of $\lambda(X)=X^{\alpha}, \alpha>0$, we show that the process $X$ should explode in finite time, say $t_{e}$, conditional on no crash.

For the case of $\lambda(X)=(\ln X)^{\alpha}$, we show that $\alpha=1$ is the borderline of two different classes of processes. We generalize the model by adding a Brownian noise and examine the blow up properties of the sample paths.
\end{abstract}

\section{Introduction}

In this paper, we introduce diffusion processes with only downward jumps with size a fixed fraction of the current process. Moreover the jump intensity is increasing as the process goes up. The motive of research of such processes is modeling of the bubble and crash phenomenon which is characterized by the rapid increase of an asset price followed by a sudden collapse (e.g., Smith et al. [19], Camerer [5], and Porter and Smith [17]). Blanchard [3] and Blanchard and Watson [4] have developed a rational expectation theory of deterministic and stochastic bubbles in discrete time.

The unrealistic features of the model, such as the exponential blow-up phenomenon in the deterministic model and the never growing phenomenon in the stochastic model, have been modified by many authors. Evans [7] proposed periodic collapsing bubbles, and Fukuta [8] developed a model with incompletely collapsing bubbles, which was generalized by Lux and Sornette [15] and Malevergne and Sornette [16]. Sornette and Anderson [20] and Anderson and Sornette [2] have studied the nonlinear rational expectation model, a modification of the Geometric Brownian Motion, under the continuous time

Received June 25, 2008; Revised July 9, 2010.

2000 Mathematics Subject Classification. 60J60, 60J45.

Key words and phrases. crash, explosion, jump diffusion.

This research was financially supported in part by Hansung University during sabbatical year (2009). 
formulation. In their papers, the crash probability increase according to the size of bubble. Our process is also a modeling of the idea that the larger the bubble size is, the greater the probability of crash seems to be. With this idea, we call downward jumps as crash.

We assume that the process is the sum of a pure jump process and its continuous compensation so that the process should be a martingale. Later, we add a Brownian noise to the process to make a diffusion process with jumps.

One of our main contributions is that, with such assumptions, the blow up curve before crash has a different nature. Indeed, for some reasonable functions $\lambda(X)$, we can figure out the expected blow up curve explicitly, and it turns out that it explodes in finite time, say $t_{e}$, depending only on the initial price, the jump intensity $\lambda(X)$, and the jump size. More precisely, for the case of $\lambda(X)=X^{\alpha}$, conditional on no crash, the process $X=X(t)$ follows

$$
X(t)=\frac{C_{1}}{\left(C_{2}-t\right)^{\frac{1}{\alpha}}}
$$

for some explicit constants $C_{1}$ and $C_{2}$. This shows that the crash should occur before $t=C_{2}$, since, if not, the process should be infinity at $t=C_{2}$. It is worth noting that this explosion comes from the nonlinearity of the process. By allowing the rate to be dependent on the process, we have extended the process to be a solution of a nonlinear stochastic differential equation.

There is another interesting result for the case of $\lambda(X)=(\ln X)^{\alpha}$. It turns out that conditional on no crash the process $X(t)$ explodes in finite time if $\alpha>1$ and grows super exponentially but never explodes in finite time if $0<\alpha<1$. Here we say that $X(t)$ grows super exponentially if $X(t) / \exp (a t) \rightarrow \infty$ for any $a<\infty$ as $t \rightarrow \infty$. We extend the above model by adding a Brownian noise and show the surprising result that, for the case of $\lambda(X)=X^{\alpha}$, there exist $\alpha$ and $\delta$ such that with positive probability the process does not explode in finite time. Moreover, the process tends to zero with positive probability. Recall that in such a case, if there is no noise, then the process explodes in finite time with probability one as in (1.1). This shows that the noise is an important factor of the crash phenomena.

This paper is organized as follows. After this introduction, in Section 2, we describe our process as a solutions of some stochastic differential equations containing jumps. Basically, we consider two types of martingale processes. Some of them will be generalized by adding a Brownian noise to the jump diffusion process. We show the existence and uniqueness results of the stochastic differential equations discussed. In Section 3, we show the exact form of the process before crash and the distribution of crash.

\section{Existence and uniqueness}

We are seeking a stochastic process which is a martingale and makes only downward jumps. One simple example is $\lambda t-N_{t}$ where $N_{t}$ is a Poisson process 
with jump intensity $\lambda$. The term $-N_{t}$ represents a pure downward jump process, where jump size is 1 and $\lambda t$ is the nondecreasing compensation making the whole process a martingale. We are interested in the process where the jump intensity depends on the state and the jump size is a fixed fraction of the state. The general version of such processes can be expressed as the solution of the following stochastic differential equation (SDE, in short), with $X_{0}>0$ :

$$
d X_{t}=\int c\left(X_{t-}, u\right)[\nu(d u d t)-\pi(d u) d t]
$$

where $c(x, u)$ is a Borel measurable function defined on $\mathbb{R}^{+} \times \mathbb{R}, \nu$ is a Poisson random measure on $\mathbb{R} \times \mathbb{R}^{+}$and $\pi$ is a $\sigma$-finite measure on $\mathbb{R}$ such that

$$
E(\nu(A \times[0, t]))=\pi(A) t
$$

for any measurable $A \subset \mathbb{R}$. Denoting $\tilde{\nu}(d u d s)=\nu(d u d s)-\pi(d u) d s$. This type of SDE is derived from the work of Skorohod [18] and Gihmann and Skorohod [9]. The solution of (2.1),

$$
X_{t}=X_{0}+\int_{0}^{t} \int c\left(X_{s-}, u\right) \tilde{\nu}(d u d s)
$$

is a martingale with jumps. For any measurable function $f$ defined on $\mathbb{R}$ such that $f(x)>0$ for $x>0$, we choose $\pi(d u)=d u$, the Lebesgue measure on $\mathbb{R}$ and

$$
c(x, u)=-\delta x 1_{(0, f(x))}(u), \quad 0<\delta<1
$$

in (2.1) to obtain a martingale process with jump rate at $x$ of $f(x)$ and jump size $-\delta x$. Here, for $x>0,1_{(0, x)}(u)=1$, if $u \in(0, x), 0$ if $u \notin(0, x)$. In this paper, we consider functions of the form

$$
f(x)=x^{\alpha}, \quad \text { or } \quad f(x)=(\ln x)^{\alpha}, \quad \alpha>0 .
$$

By this formulation, we can add endogenous variables into consideration. We now add a Brownian noise to get the SDE

$$
d X_{t}=\sigma\left(X_{t}\right) d B_{t}+b\left(X_{t}\right) d t+\int c\left(X_{t-}, u\right) \tilde{\nu}(d u d t)
$$

Now we set up mathematical background and show the existence and uniqueness of processes that we are interested in.

Let $(\Omega, \mathcal{F}, P)$ be a probability space, a non-decreasing family of $\sigma$-algebra $\mathcal{F}_{t}$ belonging to $\mathcal{F}$ be given and let $D_{\mathbb{R}}[0, \infty)$ be the set of processes on $\mathbb{R}$ which are right continuous with left limit. We consider a Poisson random measure $\nu$ on $\mathbb{R} \times \mathbb{R}^{+}$satisfying the following two properties:

(i) if $C_{1}, C_{2}, \ldots, C_{n}$ are pairwise disjoint Borel sets of $\mathbb{R} \times \mathbb{R}^{+}$, then the random variables $\nu\left(C_{1}\right), \nu\left(C_{2}\right), \ldots, \nu\left(C_{n}\right)$ are mutually independent Poisson random variables; 
(ii) for a Borel set $A \subset \mathbb{R}, \nu(A \times[0, t])$ is $\mathcal{F}_{t}$-measurable and in $D_{\mathbb{R}}[0, \infty)$, having a Poisson distribution with

$$
E(\nu(A \times[0, t]))=\pi(A) t
$$

where $\pi$ is a $\sigma$-finite measure on $\mathbb{R}$.

For a Borel set $\Delta \subset \mathbb{R}^{+}$, set

$$
\tilde{\nu}(A \times \Delta)=\nu(A \times \Delta)-\pi(A)|\Delta| .
$$

Here $|\cdot|$ is the Lebesgue measure on $\mathbb{R}$. Note that, for a fixed set $A$ with $\pi(A)<$ $\infty, \nu(A \times[0, t])$ is a Poisson process with parameter $\pi(A)$, i.e., $\tilde{\nu}(A \times[0, t])$ is a martingale.

Now for a Brownian motion starting from $0,\left\{B_{t}\right\}$ which is independent of $\nu$, consider the stochastic differential equation (2.3) where $\sigma(x), b(x)$ and $c(x, u)$ are nonrandom functions. Then, under the following Condition C, (2.3) has a unique solution, which is in $D_{\mathbb{R}}[0, \infty)$, and we call the solution 'a diffusion with jump'.

\section{Condition $\mathbf{C}$}

(C1) There is a constant $K$ such that for any $x \in \mathbb{R}$,

$$
|\sigma(x)|^{2}+|b(x)|^{2}+\int|c(x, u)|^{2} \pi(d u) \leq K\left(1+|x|^{2}\right) .
$$

(C2) (Local Lipschitz condition) For any $M>0$, there is a constant $C_{M}$ such that for $|x|<M,|y|<M$,

$$
|\sigma(x)-\sigma(y)|^{2}+|b(x)-b(y)|^{2}+\int|c(x, u)-c(y, u)|^{2} \pi(d u) \leq C_{M}|x-y|^{2} .
$$

Now, this section is devoted to prove the following Theorem 2.1 which gives the existence and uniqueness of the solution of SDE (2.4), not covered by general theory of jump diffusion above. In above setting, we let $\pi(d u)=d u$ and consider the following SDE:

$$
X_{t}=X_{0}+\int_{0}^{t} \sigma X_{s} d B_{s}+\int_{0}^{t} \int-\delta X_{s-} 1_{\left(0, X_{s-}^{\alpha}\right)}(u) \tilde{\nu}(d u d s) .
$$

Theorem 2.1. Let $\sigma \geq 0,0<\delta<1, \alpha>0$ be given and $X_{0}>0$ with $E\left(X_{0}\right)<\infty$. Then there exists a unique solution of $(2.4)$.

Proof. Let $X_{t}, Y_{t}$ be solutions of (2.4). Then

$X_{t}-Y_{t}=\int_{0}^{t} \sigma\left(X_{s}-Y_{s}\right) d B_{s}+\int_{0}^{t} \int-\delta\left[X_{s-} 1_{\left(0, X_{s-}^{\alpha}\right)}(u)-Y_{s-} 1_{\left(0, Y_{s-}^{\alpha}\right)}(u)\right] \tilde{\nu}(d u d s)$.

Take $N>0$ large, then put

$$
\begin{aligned}
& \tau_{N}=\inf \left\{t \geq 0:\left|X_{t}\right| \geq N\right\}, \\
& \tau_{N}^{\prime}=\inf \left\{t \geq 0:\left|Y_{t}\right| \geq N\right\} .
\end{aligned}
$$


Note that the coefficients do not satisfy Lipschitz conditions. Following the ideas in Theorem 3.2 of Ikeda and Watanabe [10], define a decreasing sequence of numbers $\left\{a_{n}\right\}_{n=1}^{\infty}$ such that $1=a_{0}>a_{1}>a_{2}>\cdots>a_{n}>\cdots>0$ and

$$
\int_{a_{n}}^{a_{n-1}} u^{-2} d u=n \quad \text { for } \quad n \geq 1 .
$$

Clearly, $a_{n} \rightarrow 0$ as $n \rightarrow \infty$. For each $n=1,2, \ldots$, let $\psi_{n}(u)$ be a continuous function such that its support is contained in $\left(a_{n}, a_{n-1}\right)$ and

$$
0 \leq \psi_{n}(u) \leq 2 \frac{u^{-2}}{n}, \quad \text { and } \quad \int_{a_{n}}^{a_{n-1}} \psi_{n}(u) d u=1 .
$$

Set

$$
\phi_{n}(x)=\int_{0}^{|x|} \int_{0}^{y} \psi_{n}(u) d u d y, \quad x \in \mathbb{R} .
$$

Then $\phi_{n} \in C^{2}\left(\mathbb{R}^{1}\right),\left|\phi_{n}^{\prime}(x)\right| \leq 1$ and $\phi_{n}(x) \uparrow|x|$ as $n \rightarrow \infty$. By Ito formula

$$
\begin{aligned}
& \phi_{n}\left(X_{t \wedge \tau_{N} \wedge \tau_{N}^{\prime}}-Y_{\left.t \wedge \tau_{N} \wedge \tau_{N}^{\prime}\right)}\right. \\
& =\int_{0}^{t \wedge \tau_{N} \wedge \tau_{N}^{\prime}} \sigma \phi_{n}^{\prime}\left(X_{s}-Y_{s}\right)\left(X_{s}-Y_{s}\right) d B_{s} \\
& +\frac{\sigma^{2}}{2} \int_{0}^{t \wedge \tau_{N} \wedge \tau_{N}^{\prime}} \phi_{n}^{\prime \prime}\left(X_{s}-Y_{s}\right)\left(X_{s}-Y_{s}\right)^{2} d s \\
& +\int_{0}^{t \wedge \tau_{N} \wedge \tau_{N}^{\prime}} \int\left[\phi_{n}\left(X_{s-}-Y_{s-}-\delta\left(X_{s-} 1_{\left(0, X_{s-}^{\alpha}\right)}(u)-Y_{s-} 1_{\left(0, Y_{s-}^{\alpha}\right)}(u)\right)\right)\right. \\
& \left.\quad-\phi_{n}\left(X_{s-}-Y_{s-}\right)\right] \tilde{\nu}(d u d s) \\
& +\int_{0}^{t \wedge \tau_{N} \wedge \tau_{N}^{\prime}} \int\left\{\phi _ { n } \left(X_{s-}-Y_{s-}-\delta\left(X_{s-} 1_{\left(0, X_{s-}^{\alpha}\right)}(u)-Y_{s-} 1_{\left(0, Y_{s-}^{\alpha}\right)}(u)\right)\right.\right. \\
& \quad-\phi_{n}\left(X_{s-}-Y_{s-}\right) \\
& \left.\quad+\delta \phi_{n}^{\prime}\left(X_{s-}-Y_{s-}\right)\left[X_{s-} 1_{\left(0, X_{s-}^{\alpha}\right)}(u)-Y_{s-} 1_{\left(0, Y_{s-}^{\alpha}\right)}(u)\right]\right\} d u d s .
\end{aligned}
$$

Let us denote the four integrals in (2.5) by I, II, III and IV in the order and let us estimate their expectations. For any $s \leq t \wedge \tau_{N} \wedge \tau_{N}^{\prime}, X_{s}$ and $Y_{s}$ are positive and jumps downward only and $\left|X_{s}\right| \leq N$ and $\left|Y_{s}\right| \leq N$. Therefore we can use truncation method as continuous case; for all $t$,

$$
E(\mathrm{I})=E \int_{0}^{t \wedge \tau_{N} \wedge \tau_{N}^{\prime}} \sigma \phi_{n}^{\prime}\left(X_{s}-Y_{s}\right)\left(X_{s}-Y_{s}\right) d B_{s}=0
$$

and similarly $E(\mathrm{III})=0$. By the construction of $\phi_{n}$

$$
\begin{aligned}
0 \leq E(\mathrm{II}) & \leq \frac{\sigma^{2}}{2} E \int_{0}^{t \wedge \tau_{N} \wedge \tau_{N}^{\prime}} \frac{2}{n}\left|X_{s}-Y_{s}\right|^{-2}\left|X_{s}-Y_{s}\right|^{2} d s \\
& \leq \frac{\sigma^{2} t}{n} .
\end{aligned}
$$


By the Mean Value Theorem, for some $X^{*}$ between $X_{s-}-Y_{s-}$ and

$$
X_{s-}-Y_{s-}-\delta\left(X_{s-} 1_{\left(0, X_{s-}^{\alpha}\right)}(u)-Y_{s-} 1_{\left(0, Y_{s-}^{\alpha}\right)}(u)\right)
$$

we have

IV

$$
\begin{aligned}
& =\int_{0}^{t \wedge \tau_{N} \wedge \tau_{N}^{\prime}} \int\left[-\phi_{n}^{\prime}\left(X_{s}^{*}\right)+\phi_{n}^{\prime}\left(X_{s}-Y_{s}\right)\right] \delta\left(X_{s} 1_{\left(0, X_{s}^{\alpha}\right)}(u)-Y_{s} 1_{\left(0, Y_{s}^{\alpha}\right)}(u)\right) d u d s \\
& =\delta \int_{0}^{t \wedge \tau_{N} \wedge \tau_{N}^{\prime}}\left[\phi_{n}^{\prime}\left(X_{s}-Y_{s}\right)-\phi_{n}^{\prime}\left(X_{s}^{*}\right)\right]\left(X_{s}^{1+\alpha}-Y_{s}^{1+\alpha}\right) d s .
\end{aligned}
$$

Since $\left|\phi_{n}^{\prime}(x)\right| \leq 1$ for all $x$, by the Mean Value Theorem on the function $x^{\alpha+1}$, there is $X^{* *}$ such that

$$
\begin{aligned}
|\mathrm{IV}| & \leq 2 \delta \int_{0}^{t \wedge \tau_{N} \wedge \tau_{N}^{\prime}}\left|X_{s}^{\alpha+1}-Y_{s}^{\alpha+1}\right| d s \\
& =2 \delta \int_{0}^{t \wedge \tau_{N} \wedge \tau_{N}^{\prime}}\left|(\alpha+1)\left(X_{s}^{* *}\right)^{\alpha}\left(X_{s}-Y_{s}\right)\right| d s
\end{aligned}
$$

where $X_{s}^{* *}$ lies between $X_{s}$ and $Y_{s}$ and $\left|X_{s}^{* *}\right| \leq C_{N}$ for some constant $C_{N}$ depending on $N$. Hence

$$
E(|\mathrm{IV}|) \leq \delta C_{\alpha, N} \int_{0}^{t} E\left(\left|X_{s \wedge \tau_{N} \wedge \tau_{N}^{\prime}}-Y_{s \wedge \tau_{N} \wedge \tau_{N}^{\prime}}\right|\right) d s
$$

for some constant $C_{\alpha, N}$ depending on $\alpha$ and $N$ and

$E\left[\phi_{n}\left(X_{t \wedge \tau_{N} \wedge \tau_{N}^{\prime}}-Y_{t \wedge \tau_{N} \wedge \tau_{N}^{\prime}}\right)\right] \leq \delta C_{\alpha, N} \int_{0}^{t} E\left(\left|X_{s \wedge \tau_{N} \wedge \tau_{N}^{\prime}}-Y_{s \wedge \tau_{N} \wedge \tau_{N}^{\prime}}\right|\right) d s+\frac{\sigma^{2} t}{n}$.

Therefore as $n \rightarrow \infty$,

$$
E\left[\left|X_{t \wedge \tau_{N} \wedge \tau_{N}^{\prime}}-Y_{t \wedge \tau_{N} \wedge \tau_{N}^{\prime}}\right|\right] \leq \delta C_{\alpha, N} \int_{0}^{t} E\left(\left|X_{s \wedge \tau_{N} \wedge \tau_{N}^{\prime}}-Y_{s \wedge \tau_{N} \wedge \tau_{N}^{\prime}}\right|\right) d s
$$

and by the Gronwall's Lemma and the right continuity of the processes, $X_{t}=Y_{t}$ a.s. for all $t \leq \tau_{N} \wedge \tau_{N}^{\prime}$.

For the construction of the solution, for given $N, X_{0}>0$, let

$$
\begin{aligned}
& X_{t}^{0}=X_{0}, \\
& X_{t}^{n}= \begin{cases}X_{0}+\int_{0}^{t} \sigma X_{s}^{n-1} d B_{s}-\int_{0}^{t} \int \delta X_{s-}^{n-1} 1_{\left(0,\left(X_{s-}^{n-1}\right)^{\alpha}\right)}(u) \tilde{\nu}(d u d s), \\
\quad \text { if } t \leq \tau_{N}^{n} \\
N, \quad \text { if } t \geq \tau_{N}^{n}\end{cases}
\end{aligned}
$$

where $\tau_{N}^{n}=\inf \left\{t \geq 0:\left|X_{t}^{n}\right| \geq N\right\}$. For $t \geq 0, n \geq 1$, by the construction, $X_{t}^{n} \leq N$ and $X_{t}^{n}$ is right continuous.

$$
X_{t}^{n+1}-X_{t}^{n}=\int_{0}^{t} \sigma\left(X_{s}^{n}-X_{s}^{n-1}\right) d B_{s}
$$




$$
-\int_{0}^{t} \int \delta\left(X_{s-}^{n} 1_{\left(0,\left(X_{s-}^{n}\right)^{\alpha}\right)}(u)-X_{s-}^{n-1} 1_{\left(0,\left(X_{s-}^{n-1}\right)^{\alpha}\right)}(u)\right) \tilde{\nu}(d u d s) .
$$

Apply Ito formula to $\phi_{k}(x)$, let $k \rightarrow \infty$, and use the same argument as uniqueness to get

$$
E\left|X_{t}^{n+1}-X_{t}^{n}\right| \leq \delta C_{\alpha, N} \int_{0}^{t} E\left|X_{s}^{n}-X_{s}^{n-1}\right| d s .
$$

By iteration, we have

$$
E\left|X_{t}^{n+1}-X_{t}^{n}\right| \leq \frac{\left(\delta C_{\alpha, N}\right)^{n} t^{n}}{n !}
$$

From the construction, for each $n, X_{t}^{n}$ is a martingale and right continuous with left limit. By the Doob's inequality

$$
\frac{1}{2^{n}} P\left(\sup _{0 \leq s \leq t}\left|X_{s}^{n+1}-X_{s}^{n}\right| \geq \frac{1}{2^{n}}\right) \leq E\left|X_{t}^{n+1}-X_{t}^{n}\right|,
$$

SO

$$
P\left(\sup _{0 \leq s \leq t}\left|X_{s}^{n+1}-X_{s}^{n}\right| \geq \frac{1}{2^{n}}\right) \leq \frac{\left(2 \delta C_{\alpha, N}\right)^{n} t^{n}}{n !} .
$$

Therefore by Borel-Cantelli lemma

$$
P\left(\sup _{0 \leq s \leq t}\left|X_{s}^{n+1}-X_{s}^{n}\right| \geq \frac{1}{2^{n}} \quad \text { i.o. }\right)=0 .
$$

This implies that, almost surely, $X_{s}^{n}$ converges to a limit $Y_{s}$ uniformly on $[0, t]$. Since $t>0$ is arbitrary, it follows that, almost surely, $X^{n}$ converges uniformly on any bounded time interval to a limit process $Y$. Furthermore the process $Y$ is right continuous with left limit.

Now we need to show that $Y_{t}$ is a solution of (2.4) up to $\sigma_{N}=\inf \{t \geq 0$ : $\left.\left|Y_{t}\right| \geq N\right\}$. Let

$$
Z_{t}=X_{0}+\int_{0}^{t} \sigma Y_{s} d B_{s}-\int_{0}^{t} \int \delta Y_{s-} 1_{\left(0, Y_{s-}^{\alpha}\right)}(u) \tilde{\nu}(d u d s) .
$$

Then

$$
\begin{aligned}
Z_{t}-X_{t}^{n+1}= & \int_{0}^{t} \sigma\left(Y_{s}-X_{s}^{n}\right) d B_{s} \\
& -\int_{0}^{t} \int \delta\left(Y_{s-} 1_{\left(0, Y_{s-}^{\alpha}\right)}(u)-X_{s-}^{n} 1_{\left(0,\left(X_{s-}^{n}\right)^{\alpha}\right)}(u)\right) \tilde{\nu}(d u d s) .
\end{aligned}
$$

By the same argument as uniqueness, we have

$$
E\left|Z_{t}-X_{t}^{n+1}\right| \leq \delta C_{\alpha, N} \int_{0}^{t} E\left|Y_{s}-X_{s}^{n}\right| d s
$$

and there exists $m>0$ such that $E\left|Z_{t}-X_{t}^{n+1}\right|<\epsilon$ if $n>m$ for given $\epsilon>0$. Therefore $Z_{t}$ is the pointwise limit of $X_{t}^{n}$ and $Z_{t}=Y_{t}$ a.s. Hence, let $X$ the unique solution of (2.4) up to $\tau_{N}, \tau_{0}=\inf \left\{t>0: X_{t} \leq 0\right\}$ and $X_{0}>0$. 
Then $\tau_{0}>0$ since $X_{t}$ is right continuous. Therefore by applying Ito formula to $f(x)=\ln x$ for $x>0$ before $\tau_{0}$, we have

$$
\begin{gathered}
X_{t}=X_{0} \exp \left[\sigma B_{t}-\frac{1}{2} \sigma^{2} t+\int_{0}^{t} \int \ln \left(1-\delta 1_{\left(0, X_{s-}^{\alpha}\right)}(u)\right) \tilde{\nu}(d u d s)\right. \\
\left.+\int_{0}^{t} \int\left(\ln \left(1-\delta 1_{\left(0, X_{s-}^{\alpha}\right)}(u)\right)+\delta 1_{\left(0, X_{s-}^{\alpha}\right)}(u)\right) d u d s\right] .
\end{gathered}
$$

Hence, $X_{t}>0$ if $X_{0}>0$ and $X_{t}$ is a process only with stochastic integrals. Therefore $X_{t}$ is a positive local martingale and for arbitrary $N, X_{t \wedge \tau_{N}}$ is a martingale and we have $E\left(X_{t \wedge \tau_{N}}\right)=E\left(X_{0}\right)$. Then by Fatou's Lemma, for any $t, E\left(X_{t}\right) \leq \lim _{N->\infty} E\left(X_{t \wedge \tau_{N}}\right)$. Therefore $\sup _{t} E\left[X_{t}\right]<M$ for some $M>0$. Hence $X_{t}$ does not explode in finite time a.s. and we are done.

Remark 2.1. Consider the following SDE

(2.7) $X_{t}=X_{0}+\int_{0}^{t} \sigma X_{s} d B_{s}+\int_{0}^{t} \int-\delta X_{s-} 1_{\left(X_{s-}>1\right)} 1_{\left(0,\left(\ln X_{s-}\right)^{\alpha}\right)}(u) \tilde{\nu}(d u d s)$.

The existence and uniqueness of the solution of (2.7) can be proved by a similar argument. But in this case we take

$$
\begin{aligned}
& \tau_{N, \epsilon}=\inf \left\{t>0:\left|X_{t}\right|>N \text { or }\left|X_{t}\right|<1+\epsilon\right\}, \\
& \tau_{N, \epsilon}^{\prime}=\inf \left\{t>0:\left|Y_{t}\right|>N \text { or }\left|Y_{t}\right|<1+\epsilon\right\}
\end{aligned}
$$

for $N>0$ and $\epsilon>0$. Then $X_{s-} 1_{\left(0,\left(X_{s-}\right)^{\alpha}\right)}(u)$ is changed to

$$
X_{s-} 1_{\left(X_{s-}>1\right)} 1_{\left(0,\left(\ln \left(X_{s-}\right)\right)^{\alpha}\right)}(u)
$$

and (2.5) to

$$
\begin{aligned}
\mathrm{IV} \leq & 2 \delta \int_{0}^{t \wedge \tau_{N, \epsilon} \wedge \tau_{N, \epsilon}^{\prime}} \int \mid X_{s-} 1_{\left(X_{s-}>1\right)} 1_{\left(0,\left(\ln \left(X_{s-}\right)\right)^{\alpha}\right)}(u) \\
& -Y_{s-} 1_{\left(Y_{s-}>1\right)} 1_{\left(0,\left(\ln \left(Y_{s-}\right)\right)^{\alpha}\right)}(u) \mid d u d s \\
= & 2 \delta \int_{0}^{t \wedge \tau_{N, \epsilon} \wedge \tau_{N, \epsilon}^{\prime}}\left|X_{s}\left(\ln X_{s}\right)^{\alpha}-Y_{s}\left(\ln Y_{s}\right)^{\alpha}\right| d s \\
\leq & 2 \delta C \int_{0}^{t \wedge \tau_{N, \epsilon} \wedge \tau_{N, \epsilon}^{\prime}}\left|X_{s}-Y_{s}\right| d s
\end{aligned}
$$

by the Mean Value Theorem for $f(x)=x(\ln x)^{\alpha}, f^{\prime}(x)=(\ln x)^{\alpha}+(\ln x)^{\alpha-1}$ and so $\left|f^{\prime}(x)\right| \leq C$ if $1+\epsilon<x<N$.

For the construction of the solution, let

$X_{t}^{0}=x$,

$X_{t}^{n}= \begin{cases}x+\int_{0}^{t} \sigma X_{s}^{n-1} d B_{s} & -\int_{0}^{t} \int \delta X_{s-}^{n-1} 1_{\left(X_{s-}^{n-1}>1\right)} 1_{\left(0,\left(\ln X_{s-}^{n-1}\right)^{\alpha}\right)}(u) \tilde{\nu}(d u d s), \\ & \text { if } \quad t<\tau_{N, \epsilon}^{n} \\ X_{\left(\tau_{N, \epsilon}^{n}\right)-}, & \text { if } \quad t \geq \tau_{N, \epsilon}^{n},\end{cases}$ 
where $\tau_{N, \epsilon}^{n}=\inf \left\{s \geq 0:\left|X_{s}^{n}\right| \geq N\right.$ or $\left.\left|X_{s}^{n}\right|<1+\epsilon\right\}$. Then for all $k \leq n$ and for all $t, 1+\epsilon \leq X_{t}^{k} \leq N$. Therefore by the same argument as Theorem 2.1, we have a unique solution.

\section{Crash time and its distribution}

The crash models in Section 2 consist of downward jumps and their nondecreasing compensation with or without Brownian noise. Our first interest is to explore the behavior of the compensation part of the model. If there is no crash, the process will increase. It turns out that for the case of $f(x)=x^{\alpha}, \alpha>0$, the process should explode in finite time. But then, since the jump rate tends to infinity, we conclude that the crash occurs before the time of explosion.

For $X_{0}>$, consider the equation

$$
X_{t}=X_{0}+\int_{0}^{t} \int-\delta X_{s-} 1_{\left(0, X_{s-}^{\alpha}\right)}(u) \tilde{\nu}(d u d s)
$$

which is the case with $f(x)=x^{\alpha}$ in (2.2).

Theorem 3.1. Let $X_{t}$ be a solution of (3.1). Then conditional on no jumps, $X_{t}$ is deterministic if $X_{0}$ is nonrandom, and is given by $X_{t}=\left(X_{0}^{-\alpha}-\alpha \delta t\right)^{\frac{1}{\alpha}}$. Therefore, the explosion occurs at $t_{e}=1 /\left(\alpha \delta X_{0}^{\alpha}\right)$.

Proof. Until the first jump occurs, the jump part of the process vanishes. Therefore, the process consists of only the compensation part, and it takes the form of a deterministic curve satisfying

$$
X_{t}=X_{0}+\int_{0}^{t} \delta X_{s}^{\alpha+1} d s
$$

Solving this equation, with the nonrandom initial condition $X_{0}>0$, we have a unique blow up solution. More precisely, let $\tau=\inf \left\{t>0: X_{t}-X_{t-} \neq 0\right\}$ be the first jump time. Then, conditional on $t<\tau$, the solution of (3.2) is given by

$$
X_{t}=\frac{1}{\left(X_{0}^{-\alpha}-\alpha \delta t\right)^{\frac{1}{\alpha}}} .
$$

Clearly, the explosion occurs at $t=1 /\left(\alpha \delta X_{0}^{\alpha}\right)$.

Remark 3.1. We can get Theorem 3.1 from Theorem 3.3 by letting $\sigma=0$. The reason we state this case here is that we want to show its explosion time first.

For next case, we let $f(x)=(\ln x)^{\alpha}$, then, surprisingly, it turns out that $\alpha=1$ is the borderline for processes of two different characters. Let us consider the following SDE:

$$
X_{t}=X_{0}+\int_{0}^{t} \int-\delta X_{s-} 1_{\left(X_{s-}>1\right)} 1_{\left(0,\left(\ln X_{s-}\right)^{\alpha}\right)}(u) \tilde{\nu}(d u d s) .
$$

Note that this equation is just for the case $f(x)=(\ln x)^{\alpha}$ with a small modification term $1_{\left(X_{s-}>1\right)}$. The term $1_{\left(X_{s-}>1\right)}$ is necessary to keep $\ln X_{s-}>0$. But 
it does not change our model much since, if the initial condition $X_{0}$ is much bigger than 1, even after the first jump, $X_{t}$ is also bigger than 1 . Our interest is to study the behavior of the process until the first jump. Now let $X_{t}$ be the solution of (3.3), then we have the following theorem.

Theorem 3.2. Assume $X_{0}>>1$. Conditional on no jumps, the solution of (3.3) $X_{t}$ is deterministic if $X_{0}$ is nonrandom. Moreover, we get

$$
X_{t}= \begin{cases}\left.\exp \left[(1-\alpha) \delta t+\left(\ln X_{0}\right)^{1-\alpha}\right)\right]^{\frac{1}{1-\alpha}} & \text { if } \alpha \neq 1 \\ \left(X_{0}+1\right) \exp (\exp \delta t)-1 & \text { if } \alpha=1\end{cases}
$$

Proof. By Ito formula, we have

$$
\begin{aligned}
X_{t}= & X_{0} \exp \left[\int_{0}^{t} \int \ln \left|1-1_{\left(X_{s-}>1\right)} \delta 1_{\left(0,\left(\ln X_{s-}\right)^{\alpha}\right)}(u)\right| \tilde{\nu}(d u d s)\right. \\
& +\int_{0}^{t} \int\left(\ln \left|1-1_{\left(X_{s-}>1\right)} \delta 1_{\left(0,\left(\ln X_{s-}\right)^{\alpha}\right)}(u)\right|\right. \\
& \left.\left.+1_{\left(X_{s-}>1\right)} \delta 1_{\left(0,\left(\ln X_{s-}\right)^{\alpha}\right)}(u)\right) d u d s\right],
\end{aligned}
$$

which shows that $X_{t}>0$ and (3.3) has a unique solution since it is a special case of (2.6) (see Theorem 2.1 and Remark 2.1).

Now to check the path properties of the process before the first jump, eliminate the jump part in (3.3) to get the equation

$$
\begin{aligned}
X_{t} & =X_{0}+\int_{0}^{t} \int\left(\delta X_{s} 1_{\left(X_{s-}>1\right)} 1_{\left(0,\left(\ln X_{s-}\right)^{\alpha}\right)}(u)\right) d u d s \\
& =X_{0}+\int_{0}^{t} \delta 1_{\left(X_{s}>1\right)} X_{s}\left(\ln X_{s}\right)^{\alpha} d s
\end{aligned}
$$

This is an ordinary differential equation, and, by a simple calculation with nonrandom initial condition $X_{0}>>1$, we get the following results. First, if $\alpha \neq 1$, then the solution of (3.5) is given by

$$
\left.\ln X_{t}=\left[(1-\alpha) \delta t+\left(\ln X_{0}\right)^{1-\alpha}\right)\right]^{\frac{1}{1-\alpha}} .
$$

Clearly, for $\alpha<1$, the process never explodes in finite time, but grows super exponentially with order $\exp \left[((1-\alpha) \delta t)^{\frac{1}{1-\alpha}}\right]$. On the other hand, for $\alpha>1$, it explodes at

$$
t_{e}=\frac{\left(\ln X_{0}\right)^{-\alpha+1}}{\delta(\alpha-1)} .
$$

Finally, if $\alpha=1$, the solution of $(3.5)$ is $X_{t}=X_{0} \exp (\exp \delta t)$.

As a result, if $\alpha>1$, the process cannot remain longer than $t_{e}$, similarly to Theorem 3.1. However, if $\alpha \leq 1$, the process may stay longer.

As explained above, we know from (3.4) that if $\alpha \leq 1$, then $X_{t}$ never explodes, though it grows super exponentially. But for $\alpha>1$, it explodes before

$$
t_{e}=\frac{\left(\ln X_{0}\right)^{1-\alpha}}{\delta(\alpha-1)} .
$$


This phenomenon is called a phase transition in statistical physics and has been extensively studied by many authors for a long time. At this point, we do not have clear intuition as to why $\ln x$ is the borderline of the phase transition, but we have a similar result in a different model of Jeon [11].

Now we add a Brownian noise to (3.1), that is, consider the following SDE:

$$
X_{t}=X_{0}+\int_{0}^{t} \sigma X_{s} d B_{s}+\int_{0}^{t} \int-\delta X_{s-} 1_{\left(0, X_{s-}^{\alpha}\right)}(u) \tilde{\nu}(d u d s) .
$$

Theorem 3.3. Let $X_{t}$ be a solution of (3.6). If $X_{0}>0$, then conditional on no jumps, $X_{t}$ is given by

$$
X_{t}=\exp \left(-\frac{\sigma}{2} t+B_{t}\right)^{\sigma}\left(X_{0}^{-\alpha}-\alpha \delta \int_{0}^{t}\left[\exp \left(-\frac{\sigma s}{2}+B_{s}\right)\right]^{\alpha \sigma} d s\right)^{-\frac{1}{\alpha}} .
$$

Proof. Let $\tau=\inf \left\{t>0: X_{t}-X_{t-} \neq 0\right\}$ be the first jump time. Again, before the first jump, the jump part vanishes. Then conditional on $t<\tau$, the process satisfies

$$
X_{t}=X_{0}+\int_{0}^{t} \sigma X_{s} d B_{s}+\int_{0}^{t} \delta X_{s}^{1+\alpha} d s .
$$

To solve this SDE, apply Ito formula to $f(x)=x^{-\alpha}$ and change the form as follows:

$$
d X_{t}^{-\alpha}=\left(-\alpha \delta+\frac{\sigma^{2} \alpha(\alpha+1)}{2} X_{t}^{-\alpha}\right) d t-\sigma \alpha X_{t}^{-\alpha} d B_{t}
$$

Note that the resulting SDE is linear in $X_{t}^{-\alpha}$, and the general solution of such an equation is well known. See, for example, Gihman and Skorohod ([9], p.36). By substituting all the coefficients in the general formula, we have

$$
X_{t}=\exp \left(-\frac{\sigma}{2} t+B_{t}\right)^{\sigma}\left(X_{0}^{-\alpha}-\alpha \delta \int_{0}^{t}\left[\exp \left(-\frac{\sigma s}{2}+B_{s}\right)\right]^{\alpha \sigma} d s\right)^{-\frac{1}{\alpha}}
$$

as desired.

Note that the integral

$$
\int_{0}^{t}\left[\exp \left(-\frac{\sigma s}{2}+B_{s}\right)\right]^{\alpha \sigma} d s
$$

in (3.7) is finite almost surely, since $-\sigma s / 2$ is the dominating factor. Indeed, for any $\epsilon>0$,

$$
\limsup _{s \rightarrow \infty} \frac{B_{s}}{\sqrt{2 s \log \log s}}=1
$$

almost surely, and therefore $B_{s}=o\left(s^{1 / 2+\epsilon}\right)$, a.s. Hence, if $X_{0}$ is small enough, or for a fixed $X_{0}$, if $\delta$ is small or if $\sigma$ is big enough, then, with positive probability,

$$
X_{0}^{-\alpha}>\alpha \delta \int_{0}^{t}\left[\exp \left(-\frac{\sigma s}{2}+B_{s}\right)\right]^{\alpha \sigma} d s
$$


i.e., $X_{t}$ does not explode in finite time. For such a case, with positive probability, $X_{t} \rightarrow 0$ exponentially fast, as $t \rightarrow \infty$. If $X_{t}$ becomes small, then the jump intensity also becomes small and, therefore, there may not be any crash, i.e., the bubble lands softly. It is surprising that the noise may have a great influence on the sample paths of the price. The bubble may go into crash or may make a soft landing possibly depending on the size of the volatility of the noise.

An interesting question may be posed to this endogenous modeling: What is the distribution of the first crash time. The following two theorems provide answers. Let $\tau=\inf \left\{t>0: X_{t}-X_{t-} \neq 0\right\}$ be the first jump time. We get the distribution of $\tau$ as follows.

Theorem 3.4. For the case of $f(x)=x^{\alpha}, \alpha>0$, the distribution of the crash is given by

$$
P(\tau \leq t)=1-\int_{0}^{\infty} e^{-v} \eta(d v)
$$

where

$$
\eta([0, v])=P\left(\int_{0}^{t} Z_{s}^{\alpha \sigma} d s \leq \frac{1-e^{-v \alpha \delta}}{X_{0}^{\alpha} \alpha \delta}\right)+P\left(\int_{0}^{t} Z_{s}^{\alpha \sigma} d s \geq \frac{1+e^{-v \alpha \delta}}{X_{0}^{\alpha} \alpha \delta}\right)
$$

and $Z_{t} \equiv \exp \left(-\frac{\sigma t}{2}+B_{t}\right)$.

Proof. Being $X_{t}$ a continuous process before the first crash,

$$
\begin{aligned}
P(\tau>t) & =P\left(\int_{0}^{t} \int 1_{\left(0, X_{s-}^{\alpha}\right)}(u) \nu(d u d s)=0\right) \\
& =P\left(\int_{0}^{t} \int 1_{\left(0, X_{s}^{\alpha}\right)}(u) \nu(d u d s)=0\right) .
\end{aligned}
$$

Let

$$
N_{t}=\int_{0}^{t} \int_{\left(0, X_{s}^{\alpha}\right)}(u) \nu(d u d s) .
$$

Then $\nu$ and $\left\{X_{s}\right\}$ are independent and $N_{t}=\nu(A(t))$, where

$$
A(t)=\left\{(u, s): 0 \leq u \leq X_{s}^{\alpha}, 0 \leq s \leq t\right\} .
$$

That is, $N_{t}$ is a Poisson point process with intensity

$$
\int_{0}^{t} \int_{A(s)}(u, s) d u d s=\int_{0}^{t} X_{s}^{\alpha} d s
$$

i.e., $N_{t}-\int_{0}^{t} X_{s}^{\alpha} d s$ is a jump martingale with jump size 1 . Therefore if we let $\beta(t)$ be as $\int_{0}^{\beta(t)} X_{s}^{\alpha} d s=t$, then $N_{\beta(t)}$ is a Poisson process such that $N_{\beta(t)}-t$ is a martingale. Therefore, $V_{t} \equiv N_{\beta(t)}$ is a Poisson process with intensity $t$. Consequently,

$$
\begin{aligned}
P(\tau>t) & =P\left(N_{r}=0 \quad \text { for all } r \leq t\right) \\
& =P\left(N_{\beta(r)}=0 \text { for all } r \leq \beta^{-1}(t)\right)
\end{aligned}
$$




$$
\begin{aligned}
& =P\left(V_{r}=0 \text { for all } r \leq \beta^{-1}(t)\right) \\
& =\int_{0}^{\infty} P\left(V_{r}=0 \text { for all } r \leq \int_{0}^{t} X_{s}^{\alpha} d s \mid \int_{0}^{t} X_{s}^{\alpha} d s=v\right) \eta(d v) \\
& =\int_{0}^{\infty} e^{-v} \eta(d v),
\end{aligned}
$$

where $\eta(\cdot)=P\left(\int_{0}^{t} X_{s}^{\alpha} d s \in \cdot\right)$ and $X_{t}$ is given by (3.7). So

$$
\begin{aligned}
P(\tau \leq t) & =1-P(\tau>t) \\
& =1-\int_{0}^{\infty} e^{-v} \eta(d v) .
\end{aligned}
$$

We write $Z_{t}=\exp \left(-\frac{\sigma t}{2}+B_{t}\right)$ for notational simplicity. Then since

$$
X_{t}^{\alpha}=-\frac{1}{\alpha \delta} \cdot \frac{\frac{d}{d t}\left(X_{0}^{-\alpha}-\alpha \delta \int_{0}^{t} Z_{s}^{\alpha \sigma} d s\right)}{X_{0}^{-\alpha}-\alpha \delta \int_{0}^{t} Z_{s}^{\alpha \sigma} d s}
$$

we have

$$
\int_{0}^{t} X_{s}^{\alpha} d s=\ln \left|1-\alpha \delta X_{0}^{\alpha} \int_{0}^{t} Z_{s}^{\alpha \sigma} d s\right|^{-\frac{1}{\alpha \delta}} .
$$

Hence

$$
\begin{aligned}
\eta([0, v]) & =P\left(\int_{0}^{t} X_{s}^{\alpha} d s \leq v\right) \\
& =P\left(\left|1-\alpha \delta X_{0}^{\alpha} \int_{0}^{t} Z_{s}^{\alpha \sigma} d s\right| \geq e^{-v \alpha \delta}\right) \\
& =P\left(\int_{0}^{t} Z_{s}^{\alpha \sigma} d s \leq \frac{1-e^{-v \alpha \delta}}{X_{0}^{\alpha} \alpha \delta}, \text { or } \int_{0}^{t} Z_{s}^{\alpha \sigma} d s \geq \frac{1+e^{-v \alpha \delta}}{X_{0}^{\alpha} \alpha \delta}\right) \\
& =P\left(\int_{0}^{t} Z_{s}^{\alpha \sigma} d s \leq \frac{1-e^{-v \alpha \delta}}{X_{0}^{\alpha} \alpha \delta}\right)+P\left(\int_{0}^{t} Z_{s}^{\alpha \sigma} d s \geq \frac{1+e^{-v \alpha \delta}}{X_{0}^{\alpha} \alpha \delta}\right),
\end{aligned}
$$

which completes the proof.

Remark 3.2. For the case of $f(x)=x^{\alpha}, \alpha>0$, without noise, the distribution of the crash is given by

$$
P(\tau \leq t)=1-\left|1-\alpha \delta X_{0}^{\alpha} t\right|^{\frac{1}{\alpha \delta}} \quad\left(t<\frac{1}{\alpha \delta X_{0}^{\alpha}}\right) .
$$

This is a special case of Theorem 3.4, but it is worth to mention.

By the definition of $\beta(t)$ in the proof of Theorem 3.4,

$$
\begin{aligned}
\beta^{-1}(t) & =\int_{0}^{t} X_{s}^{\alpha} d s \\
& =\int_{0}^{t} \frac{d s}{X_{0}^{-\alpha}-\alpha \delta s}
\end{aligned}
$$




$$
=\ln \left|1-\alpha \delta X_{0}^{\alpha} t\right|^{-\frac{1}{\alpha \delta}} .
$$

Therefore

$$
P(\tau>t)=\exp \left[-\ln \left|1-\alpha \delta X_{0}^{\alpha} t\right|^{-\frac{1}{\alpha \delta}}\right]=\left|1-\alpha \delta X_{0}^{\alpha} t\right|^{\frac{1}{\alpha \delta}}
$$

and (3.8) follows. One interesting result of this is that the distribution has a different form around $\alpha \delta=1$. Indeed, the density function, the derivative of (3.8), blows up to infinity if $\alpha \delta>1$, while it decreases to 0 if $\alpha \delta<1$ as $t \rightarrow t_{e}$.

\section{References}

[1] M. C. Adam and A. Szafarz, Speculative bubbles and financial markets, Oxford Econmic Papers 44 (1992), no. 4, 626-640.

[2] J. V. Andersen and D. Sornette, Fearless versus fearful speculative financial bubbles, Phys. A 337 (2004), no. 3-4, 565-585.

[3] O. J. Blanchard, Speculative bubbles, crashes and rational expectations, Economic Letters 3 (1979), 387-389.

[4] O. J. Blanchard and M. W. Watson, Bubbles, Rational Expectations and Speculative Markets, in: P. Wachtel, eds., Crisis in Economic and Financial Structure: Bubbles, Bursts, and Shocks. Lexington Books: Lexington, 1982.

[5] C. Camerer, Bubbles and fads in asset prices, Journal of Economic Surveys 3 (1989), no. $1,3-41$.

[6] K. L. Chung and R. J. Williams, Introduction to Stochastic Integration, Second edition. Probability and its Applications. Birkhäuser Boston, Inc., Boston, MA, 1990.

[7] G. W. Evans, Pitfalls in testing for explosive bubbles in asset prices, American Economic Review 81 (1991), 922-930.

[8] Y. Fukuta, A simple discrete-time approximation of continuous-time bubbles, J. Econom. Dynam. Control 22 (1998), no. 6, 937-954.

[9] I. I. Gihman and A. V. Skorohod, Stochastic Differential Equations, Springer-Verlag, New York-Heidelberg, 1972.

[10] N. Ikeda and S. Watanabe, Stochastic Differential Equations and Diffusion Processes, Second edition. North-Holland Mathematical Library, 24. North-Holland Publishing Co., Amsterdam; Kodansha, Ltd., Tokyo, 1989.

[11] I. Jeon, Stochastic fragmentation and some sufficient conditions for shattering transition, J. Korean Math. Soc. 39 (2002), no. 4, 543-558.

[12] A. Johansen, O. Ledoit, and D. Sornette, Crashes as critical points, Int. J. Theo. \& Appl. Finance 3 (2000), no. 2, 219-255.

[13] A. Johansen and D. Sornette, Critical crashes, Risk 12 (1999), no. 1, 91-95.

[14] I. Karatzas and S. E. Shreve, Brownian Motion and Stochastic Calculus, SpringerVerlag, New York, 1988.

[15] T. Lux and D. Sornette, On rational bubbles and fat tails, Journal of Money, Credit, and Banking 34 (2002), no. 3, 589-610.

[16] Y. Malevergne and D. Sornette, Multi-dimensional rational bubbles and fat tails, Quantitative Finance 1 (2001), 533-541.

[17] D. Porter and V. Smith, Stock market bubbles in the laboratory, Applied Mathematical Finance (1994), 111-127.

[18] A. V. Skorohod, Studies in The Theory of Random Processes, Inc. Addison-Wesley Publishing Co., Inc., Reading, Mass., 1965.

[19] V. Smith, G. Suchanek, and A. Williams, Bubbles crashes, and endogenous expectations in experimental stock asset markets, Econometrica 56 (1988), 1119-1151.

[20] D. Sornette and J. V. Andersen, A nonlinear super-exponential rational model of speculative financial bubbles, Int. J. Mod. Phys. C 13 (2002), no. 2, 171-188. 
YoungmeE Kwon

Department of Multimedia

HANSUNG UNIVERSITY

Seoul 136-792, Korea

E-mail address: ymkwon@hansung.ac.kr

INTAE JEON

Department of Mathematics

Catholic University of Korea

BuCHEON 420-743, KoreA

E-mail address: injeon@cuk.ac.kr

Hye-JeONG KANG

Department of Mathematics

Seoul National University

Seoul 151-742, Korea

E-mail address: hjkang@snu.ac.kr 